\title{
OBSERVATIONS OF TWO DIFFERENT CLUSTER TYPES IN THE LMC
}

\author{
P. LINDE, G. LYNGA \\ Lund Observatory \\ Box 43, S-221 00 Lund \\ Sweden \\ B.E. WESTERLUND \\ Uppsala Observatory \\ Box 515, S-751 20 Uppsala \\ Sweden
}

\begin{abstract}
The aim of our project is to study the star-formation history in open clusters and field stars in two regions on either side of the bar of the Large Magellanic Cloud (LMC). In a forthcoming paper we shall describe the observations and the characteristics of the clusters. Here, we summarise the results of the BV photometry of three of the cluster fields: LW $55\left(4^{\mathrm{h}} 46 . \mathrm{m} 6,-73^{\circ} 30^{\prime}\right)$, HS $96\left(5^{\mathrm{h}} 03 .^{\mathrm{m}} 4,-65^{\circ} 57^{\prime}\right)$ and SL $196\left(5^{\mathrm{h}} 03 .{ }^{\mathrm{m}} 6,-65^{\circ} 54^{\prime}\right.$, epoch 1950$)$. They were all observed with an RCA CCD at the $2.2-\mathrm{m}$ telescope at La Silla.
\end{abstract}

\section{Results}

HS 96 (Fig. 1a) appears to have a main sequence reaching about $\mathrm{V}=16.5$. It may be followed for about $5 \mathrm{mag}$. There are no red giants in the cluster. Its age can be estimated to $0.2 \mathrm{Gyr}$ or less, while the stellar population in its surroundings (Fig. 1b) is slightly older.

The main sequence of SL 196 (Fig. 2a) ends at about $\mathrm{V}=18$. A group of red giants is seen at about $\mathrm{V}=18.2, \mathrm{~B}-\mathrm{V}=0.8$, but as the field near the cluster (Fig. 2b) is extremely rich in both blue and red stars, it is difficult to define the cluster accurately.

The surrounding field of SL 196 has a population very similar to that of the cluster itself, and we may conclude that they represent a population of about 0.3 Gyr. The cluster HS 96 is very close to SL 196 on the sky but is slightly younger. It appears important to determine whether the clusters are due to a triggered star formation process in the same star-burst or if they originate in two different bursts.

A comparison of the cluster LW 55 (Fig. 3a) with its surroundings (Fig. 3b) outside 30 arcsec shows that the LW 55 main sequence ends at about $V=19$. Its red giant clump is found at about $\mathrm{V}=19, \mathrm{~B}-\mathrm{V}=0.8$. This is slightly above the giant clump of the surrounding field. It may be explained either as a metallicity effect - the cluster may be slightly older than the surrounding field and thus of lower metallicity - or as a reddening effect. LW 55 is apparently one of the many clusters in the 2-3 Gyr generation.

It is of interest to note the similarity in age between the clusters and their surrounding field populations. The young Northern field and its clusters are about 0.2-0.3 Gyr old, whereas the age of the Southern field and LW 55 is about 2-3 Gyr. Possible scenarios indicated by all the observations will be discussed later. 


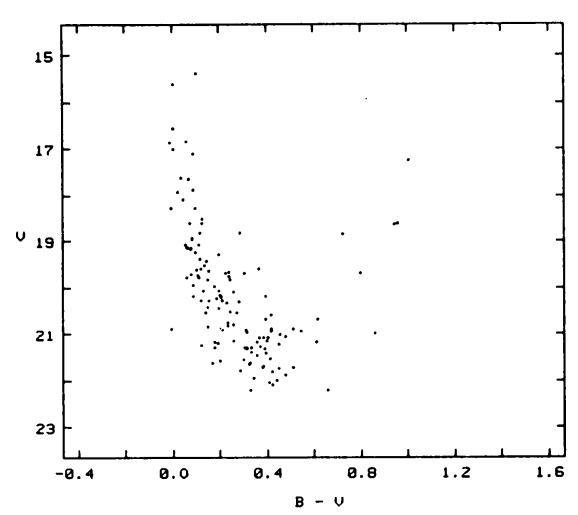

Figure 1a. CMD for cluster HS 96 inside a radius of 30 seconds of arc.

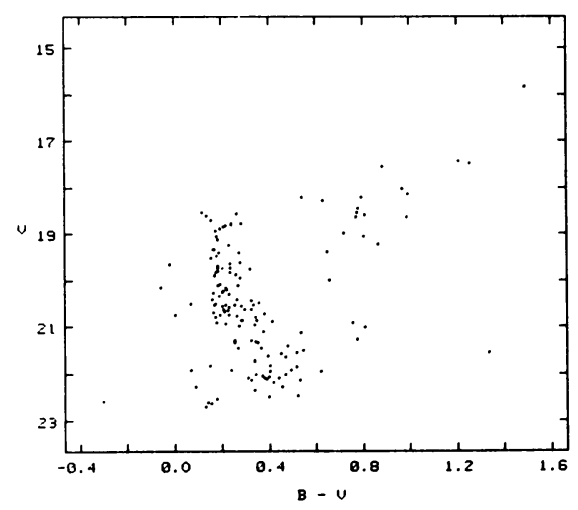

Figure 2a. CMD for cluster SL 196 inside a radius of 23 seconds of arc.

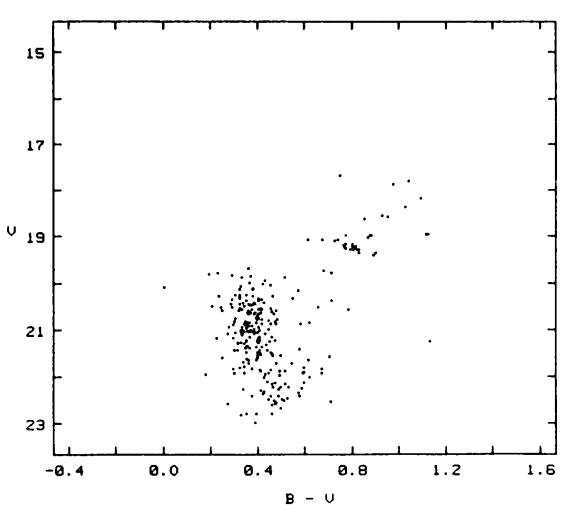

Figure 3a. CMD for cluster LW 55 inside a radius of 30 seconds of arc.

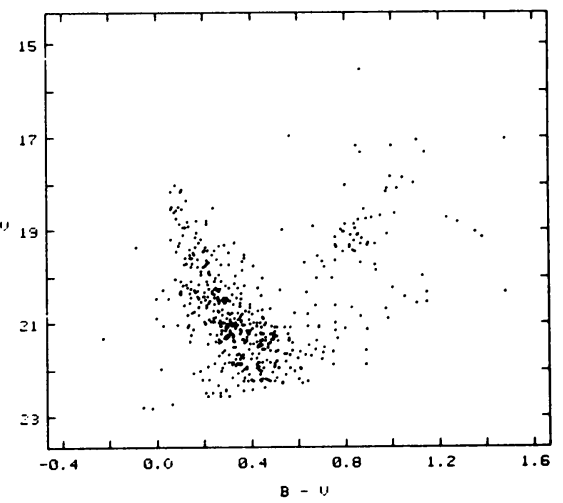

Figure 1b. CMD for the surroundings of cluster HS 96, outside a radius of 30 seconds of arc.

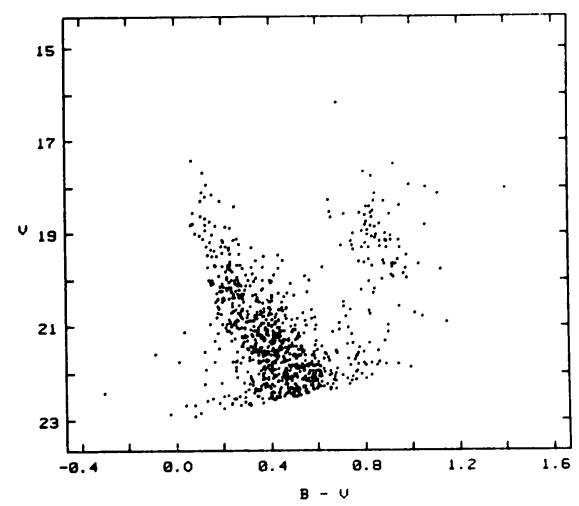

Figure 2b. CMD for the surroundings of cluster SL 196, outside a radius of 23 seconds of arc.

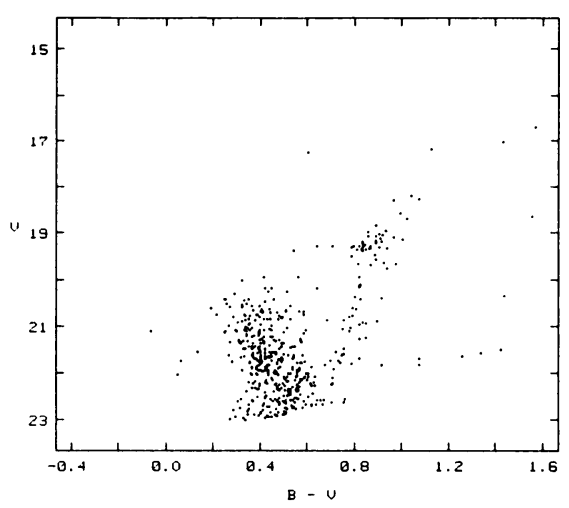

Figure 3b. CMD for the surroundings of cluster LW 55, outside a radius of 30 seconds of arc. 